\title{
Annual Report of the Korea Central Cancer Registry Program 2000: Based on Registered Data from 131 Hospitals
}

\author{
Jong-Myon Bae, M.D., Young-Joo Won, M.R.A., Kyu-Won Jung, M.S. and Jae-Gahb Park, M.D. \\ Headquarters of Korea Central Cancer Registry, National Cancer Center, Goyang, Korea
}

Purpose: The Korea Central Cancer Registry (KCCR) program completed a nationwide hospital-based cancer registry to provide basic statistical data on cancer incidence.

Materials and Methods: In 2000, 131 hospitals participated in the KCCR program. All cancer registry data, which were submitted by the participating hospitals during the year, were reviewed and analyzed by the committee members.

Results: Of the 94,474 cases that were registered, 7,735 $(8.2 \%)$ duplication cases were excluded. Of the remaining 86,739 cases, 2,893 cases $(3.3 \%)$ of carcinoma in situ (morphology code/2) were excluded, leaving a final total of 83,846 cases for analysis, $48,005(57.3 \%)$ male and $35,841(42.7 \%)$ female. The leading age group was $60 \sim 64$

\section{INTRODUCTION}

The Korea Central Cancer Registry (KCCR) is a nationwide hospital-based cancer registry, begun in 1980 (1). After "The Korea Cancer Control Workshop", held in 1978 with the aid of WHO, the Minister of Health and Welfare merged the various individual cancer registry programs into a single, nationwide, hospital-based program. The purpose was to accurately measure the cancer incidence in the nation and to construct useful databases for basic cancer research studies and treatment plans. Twenty annual reports and two 5-year reports (July 1982-June 1987, July 1988-June 1992) had been published by May 2001 (2). The Headquarters of KCCR moved to the National Cancer Center in September 2000. The central committee of KCCR is composed of a chief, an executive secretary, a medical record administrator, a statistician, and 11 advisory members.

The aim of this paper is to provide a summary of the $21^{\text {st }}$ annual report of the KCCR program, which was published in

Correspondence: Jong-Myon Bae, Cancer Registration \& Biostatistics Branch National Cancer, Goyang-si, Gyeonggi-do 411764, Korea. (Tel) 82-31-920-2131, (Fax) 82-31-920-2139, (E-mail) canreg@korea.com

This study was supported by a grant of the 2000 Korea Central Registry program, Ministry of Health \& Welfare, R.O.K years $(15.1 \%)$. The six leading primary cancer sites in the order of their relative frequency were the stomach $(20.8 \%)$, lung $(12.2 \%)$, liver $(12.2 \%)$, colorectum $(10.3 \%)$, breast $(6.5 \%)$, and uterine cervix $(4.5 \%)$. In males, the three leading primary cancer sites were the stomach $(24.5 \%)$, liver $(16.3 \%)$, and lung $(16.3 \%)$. In females, the stomach $(15.8 \%)$, breast $(15.1 \%)$, and uterine cervix $(10.6 \%)$. Among the 1,126 cases of childhood malignancies, leukemia $(32.7 \%)$ was the most common cancer type.

Conclusion: We report our analysis of the KCCR data from 131 nationwide hospitals during 2000. (Cancer $R e$ search and Treatment 2002;34:77-83)

Key Words: Neoplasms, Registries, Annual reports

22 February 2002 (3). This $21^{\text {st }}$ annual report contains information on the relative frequencies of malignant neoplasms in the Republic of Korea, and was derived from the database of the nationwide, hospital-based cancer registry program comprised of data collected between January 1, 2000 and December 31,2000 .

\section{METHODS}

Since 1992 the annual reports have shown the results of the data analysis from January to December of the preceding year, affording an easy comparison between the years. From 1993, all the data have been statistically analyzed by a PC program, which was constructed by the KCCR committee. In 1995, a PC software program for the cancer registry was developed and distributed to all participating hospitals after a training period for medical record administrators. The participating hospitals have sent their data in the form of diskettes since 1996. In 1996, the second edition of the International Classification of Diseases for Oncology (ICD-O, 2nd edition) was translated into Korean and distributed to all the participating hospitals. Topography and Morphology codes of the ICD-O, 2nd edition were used as extracted from the 16th annual report. On May $6^{\text {th }} 1998$, "In-service Training Workshop for Cancer Registry" was held to establish an efficient conduct of the cancer registry.

Among 170 registered hospitals, 131 participated in the KCCR program in 2000. All cancer registry data submitted by the participating hospitals on diskettes during the year were 
reviewed and analyzed by the committee members who were all Board-qualified clinical oncologists and pathologists. After correction of an erroneous coding of topography and morphology in the ICD-O2, the data were entered for computer analysis. To avoid duplication, the computer compared the resident registration number of all subjects. Much emphasis was placed on the diagnosis during this selection procedure. Cases diagnosed by histological examination were preferentially selected for inclusion. Of 94,474 registered malignancies, 7,735 $(8.2 \%)$ duplicated malignancies were excluded. Of the remaining 86,739 malignancies, 2,893 malignancies (3.3\%) of carcinoma in situ (Morphology code/2) were also excluded, leaving a total of 83,846 malignancies for final analysis. The advisory committee reevaluated the analysis results. The report of the KCCR program was published and released annually by the Ministry of Health and Welfare.

\section{RESULTS}

1) Marginal frequency of malignant neoplasm and carcinoma in situ by age, sex, and topography (Table 1)

Of the total of 83,846 registered malignancies, 48,005 $(57.3 \%)$ were male and $35,841(42.7 \%)$ were female; a sex ratio of 1.34. The most common age groups in the order of their relative frequency were $60 \sim 64$ years $(15.1 \%), 55 \sim 59(13.4 \%)$, $65 \sim 69$ (13.3\%), 70 74 (10.2\%), 50 54 (10.2\%), and 45 49 $(8.4 \%)$. The relative frequencies of the age groups below 45 were $0.1 \sim 7.5 \%$. The cumulative frequency of the age groups above 75 was $9.5 \%$. The leading primary cancer sites in the order of their relative frequency were the stomach $(20.8 \%)$, bronchus \& lung $(12.2 \%)$, liver \& intrahepatic bile ducts $(12.2 \%)$, colorectum $(10.3 \%)$, breast $(6.5 \%)$, uterine cervix $(4.5 \%)$, thyroid $(3.5 \%)$, hematopoietic \& reticuloendothelial systems $(2.8 \%)$, urinary bladder $(2.4 \%)$, and pancreas $(2.3 \%)$ (Fig. 1).

In males, the leading primary cancer sites were the stomach (24.5\%), liver \& intrahepatic bile ducts (16.3\%), bronchus \& lung $(16.3 \%)$, colorectum $(10.2 \%)$, urinary bladder $(3.5 \%)$, esophagus $(2.9 \%)$, hematopoietic \& reticuloendothelial systems $(2.7 \%)$, pancreas $(2.3 \%)$, prostate $(2.2 \%)$, and kidney $(1.9 \%)$.
In females, the stomach $(15.8 \%)$ was the most common site, followed by the breast $(15.1 \%)$, uterine cervix (10.6\%), colorectum $(10.5 \%)$, thyroid $(6.9 \%)$, bronchus \& lung $(6.8 \%)$, liver $\&$ intrahepatic bile ducts $(6.7 \%)$, ovary $(4.1 \%)$, hematopoietic $\&$ reticuloendothelial systems $(2.9 \%)$, and pancreas $(2.2 \%)$.

In the total of 2,893 cases of carcinoma in situ, 108 (3.7\%) were male and 2,785 (96.3\%) were female (Table 2). Among these females, the major cancer sites were the uterine cervix [2,327 cases (83.6\%)] and breast [369 cases (13.2\%)], which composed $38.0 \%$ of the registered uterine cervix cancer cases and $6.4 \%$ of the registered female breast cancer cases, respectively.

\section{2) Distribution by province}

The leading metropolitan areas and regional provinces in the order of their relative frequency of registered malignancies were Seoul city (23.2\%), Gyeonggido (17.6\%), Busan city (8.5\%), Gyeongsangbugdo (6.6\%), Gyeongsangnamdo (6.3\%), Daegu city $(5.0 \%)$, Incheon city (4.9\%), Chungcheongnamdo (4.8\%), Jeonlanamdo (4.5\%), Jeonlabugdo (4.3\%), Gangweondo (3.8\%), Chungcheongbugdo (3.5\%), Daejeon city (2.5\%), Gwangju city $(2.2 \%)$, Ulsan city $(1.4 \%)$ and Jejudo $(0.7 \%)$. These results

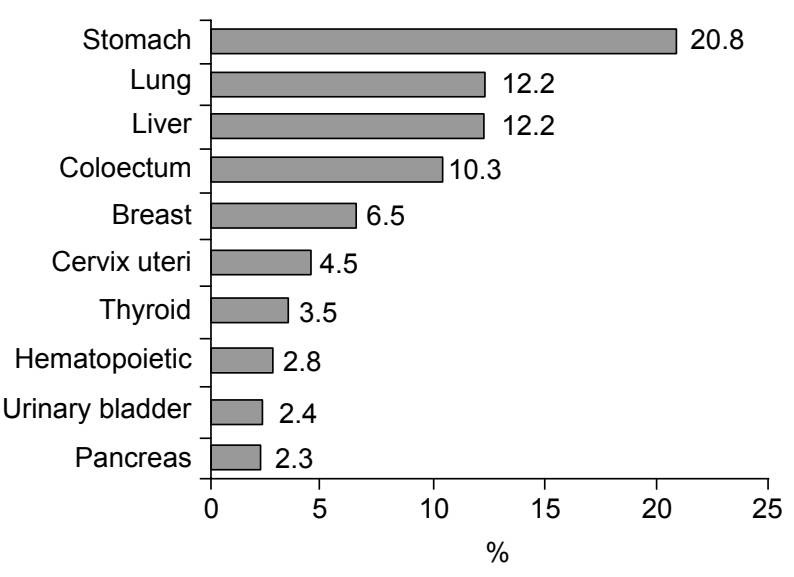

Fig. 1. Ten leading primary cancer sites in total registered cases.

Table 1. Ten leading primary cancer sites by sex

\begin{tabular}{|c|c|c|c|c|c|c|}
\hline & \multicolumn{2}{|c|}{ Total $(\mathrm{n}=83,846)$} & \multicolumn{2}{|c|}{ Male $(\mathrm{n}=48,005)$} & \multicolumn{2}{|c|}{ Female $(n=35,841)$} \\
\hline & Organ & Cases $(\%)$ & Organ & Cases $(\%)$ & Organ & Cases $(\%)$ \\
\hline 1 & Stomach & $17,439(20.8)$ & Stomach & $11,761(24.5)$ & Stomach & $5,678(15.8)$ \\
\hline 2 & Lung & $10,230(12.2)$ & Liver & $7,825(16.3)$ & Breast & $5,409(15.1)$ \\
\hline 3 & Liver & $10,214(12.2)$ & Lung & $7,809(16.3)$ & Cervix uteri & $3,803(10.6)$ \\
\hline 4 & Colorectum & $8,648(10.3)$ & Colorectum & $4,897(10.2)$ & Colorectum & $3,751(10.5)$ \\
\hline 5 & Breast & $5,444(6.5)$ & Bladder & $1,666(3.5)$ & Thyroid & $2,469(6.9)$ \\
\hline 6 & Cervix uteri & $3,803(4.5)$ & Esophagus & $1,394(2.9)$ & Lung & $2,421(6.8)$ \\
\hline 7 & Thyroid & $2,921(3.5)$ & Hematopoietics & $1,320(2.7)$ & Liver & $2,389(6.7)$ \\
\hline 8 & Hematopoietics & $2,366(2.8)$ & Pancreas & $1,120(2.3)$ & Ovary & $1,480(4.1)$ \\
\hline 9 & Urinary bladder & $2,045(2.4)$ & Prostate & $1,057(2.2)$ & Hematopoietics & $1,046(2.9)$ \\
\hline 10 & Pancreas & $1,894(2.3)$ & Kidney & 891 (1.9) & Pancreas & $774(2.2)$ \\
\hline
\end{tabular}


Table 2. Frequency of in situ cases

\begin{tabular}{lllllr}
\hline & \multicolumn{2}{c}{ Male $(\mathrm{n}=108)$} & & \multicolumn{2}{c}{ Female $(\mathrm{n}=2,785)$} \\
\cline { 6 - 6 } \cline { 5 - 6 } & Organ & Cases $(\%)$ & & \multicolumn{1}{c}{ Organ } & Cases $(\%)$ \\
\hline 1 & Bladder & $21(19.4)$ & & Cervix uteri & $2,327(83.6)$ \\
2 & Stomach & $14(13.0)$ & & Breast & $369(13.2)$ \\
3 & Colon & $11(10.2)$ & & Vulva & $18(0.6)$ \\
4 & Larynx & $10(9.3)$ & & Skin & $13(0.5)$ \\
5 & Skin & $9(8.3)$ & & Uterus, NOS & $5(0.2)$ \\
etc. & & $43(39.8)$ & & $53(1.9)$ \\
\hline
\end{tabular}

Table 3. Distribution of the diagnosis methods

\begin{tabular}{lccc}
\hline \multicolumn{1}{c}{ Diagnosis methods } & Total (\%) & Male (\%) & Female (\%) \\
\hline Microscopic test* & 82.1 & 79.2 & 85.9 \\
Clinical only & 2.1 & 2.1 & 2.2 \\
Clinical Investigation & 15.2 & 18.1 & 11.3 \\
Exploratory surgery & 0.2 & 0.2 & 0.2 \\
Specific biochemical, & 0.5 & 0.5 & 0.5 \\
$\quad$ immunological test & & & \\
\hline
\end{tabular}

*by cytology, hematology, histology, exploratory surgery and autopsy with histologic exam.

Table 4. Frequency of topography and morphology in primary cancer sites

\begin{tabular}{|c|c|c|c|c|c|c|c|c|}
\hline & T-code & Topography & Cases & $\%$ & M-code & Morphology & Cases & $\%$ \\
\hline \multirow{6}{*}{$\begin{array}{l}\text { Stomach } \\
\text { (C16) }\end{array}$} & $\mathrm{C} 16.3$ & Gastric antrum & 5677 & 32.6 & 814 & Adenocarcinoma & 8470 & 48.6 \\
\hline & C16.2 & Body & 3634 & 20.8 & 821 & Tubular adeno ca. & 3840 & 22.0 \\
\hline & $\mathrm{C} 16.8$ & Overlapping lesion & 2441 & 14.0 & 849 & Signet ring cell ca. & 2702 & 15.5 \\
\hline & $\mathrm{C} 16.0$ & Cardia, NOS & 621 & 3.6 & 848 & Mucinous adeno ca. & 287 & 1.6 \\
\hline & C16.5 & Lesser curvature & 435 & 2.5 & 826 & Papillary adeno ca. & 149 & 0.9 \\
\hline & etc. & & 4631 & 26.5 & etc. & & 1991 & 11.4 \\
\hline \multirow{6}{*}{$\begin{array}{l}\text { Lung } \\
\text { (C34) }\end{array}$} & C34.1 & Upper lung & 2921 & 28.6 & 807 & Squamous cell ca. & 3371 & 33.0 \\
\hline & C34.3 & Lower lung & 2109 & 20.6 & 814 & Adenocarcinoma & 2625 & 25.7 \\
\hline & C34.0 & Main bronchus & 458 & 4.5 & 804 & Small cell ca. & 1363 & 13.3 \\
\hline & C34.2 & Middle lobe & 340 & 3.3 & 825 & $\begin{array}{l}\text { Bronchiolo-alveolar } \\
\text { adenocarcinoma }\end{array}$ & 152 & 1.5 \\
\hline & C34.8 & Overlapping lesion & 209 & 2.0 & 824 & Carcinoid tumor & 65 & 0.6 \\
\hline & C34.9 & Lung, NOS & 4193 & 41.0 & etc. & & 2654 & 25.9 \\
\hline Liver & $\mathrm{C} 22.0$ & Liver & 8591 & 84.1 & 817 & Hepatocellular ca. & 7262 & 71.1 \\
\hline \multirow[t]{6}{*}{ (C22) } & $\mathrm{C} 22.1$ & Intrahepatic bile duct & 1623 & 15.9 & 816 & Cholangiocarcinoma & 553 & 5.4 \\
\hline & & & & & 814 & Adenocarcinoma & 293 & 2.9 \\
\hline & & & & & 897 & Hepatoblastoma & 25 & 0.2 \\
\hline & & & & & 818 & $\begin{array}{l}\text { Combined hepatocellular ca } \\
\text { and cholangio ca. }\end{array}$ & 17 & 0.2 \\
\hline & & & & & 821 & Tubular adeno ca. & 17 & 0.2 \\
\hline & & & & & etc. & & 2047 & 20.0 \\
\hline Breast & C50.4 & Upper outer & 1742 & 32.0 & 850 & Infiltrating duct ca. & 4348 & 79.9 \\
\hline \multirow[t]{5}{*}{$(\mathrm{C} 50)$} & C50.8 & Overlapping lesion & 658 & 12.1 & 852 & Lobular ca. & 193 & 3.5 \\
\hline & $\mathrm{C} 50.2$ & Upper inner & 586 & 10.8 & 848 & Mucinous adeno ca. & 122 & 2.2 \\
\hline & C50.5 & Lower outer & 306 & 5.6 & 851 & Medullary ca. & 108 & 2.0 \\
\hline & C50.1 & Central portion & 212 & 3.9 & 805 & Papillary ca. & 54 & 1.0 \\
\hline & etc. & & 1940 & 35.6 & etc. & & 619 & 11.4 \\
\hline Rectum & C20.9 & Rectum, NOS & 3997 & 88.9 & 814 & Adenocarcinoma & 2774 & 61.7 \\
\hline \multirow[t]{5}{*}{$(\mathrm{C} 19 \sim \mathrm{C} 20)$} & C19.9 & Rectosigmoid junction & 499 & 11.1 & 821 & Tubular adeno ca. & 940 & 20.9 \\
\hline & & & & & 848 & Mucinous adeno ca. & 164 & 3.6 \\
\hline & & & & & 826 & Papillary adeno ca. & 92 & 2.0 \\
\hline & & & & & 824 & Carcinoid tumor & 50 & 1.1 \\
\hline & & & & & etc. & & 476 & 10.6 \\
\hline Colon & C18.7 & Sigmoid colon & 1579 & 38.0 & 814 & Adenocarcinoma & 2513 & 60.5 \\
\hline \multirow[t]{5}{*}{ (C18) } & $\mathrm{C} 18.2$ & Ascending colon & 790 & 19.0 & 821 & Tubular adenocarcinoma & 774 & 18.6 \\
\hline & $\mathrm{C} 18.4$ & Transverse colon & 300 & 7.2 & 848 & Mucinous adenocarcinoma & 243 & 5.9 \\
\hline & $\mathrm{C} 18.0$ & Cecum & 252 & 6.1 & 826 & Papillary adenocarcinoma & 54 & 1.3 \\
\hline & C18.6 & Descending colon & 204 & 4.9 & 849 & $\begin{array}{l}\text { Malignant lymphoma, large cell, } \\
\text { diffuse, specified type }\end{array}$ & 36 & 0.9 \\
\hline & etc. & & 1,027 & 24.7 & etc. & & 532 & 12.8 \\
\hline Cervix & C53.9 & Cervix uteri & 3633 & 95.5 & 807 & Squamous cell ca. & 2928 & 77.0 \\
\hline \multirow[t]{5}{*}{ uteri (C53) } & C53.1 & Exocervix & 106 & 2.8 & 814 & Adenocarcinoma & 282 & 7.4 \\
\hline & C53.0 & Endocervix & 52 & 1.4 & 856 & Adenosquamous ca. & 83 & 2.2 \\
\hline & C53.8 & Overlapping lesion & 12 & 0.3 & 805 & Papillary carcinoma & 26 & 0.7 \\
\hline & & & & & 821 & Tublar adeno ca. & 17 & 0.4 \\
\hline & & & & & etc. & & 467 & 12.3 \\
\hline
\end{tabular}


Table 5. Distribution of childhood malignancies

\begin{tabular}{|c|c|c|c|c|c|c|}
\hline & \multicolumn{2}{|c|}{ Total $(n=1,126)$} & \multicolumn{2}{|c|}{ Boy $(n=596)$} & \multicolumn{2}{|c|}{ Girl $(n=530)$} \\
\hline & Organ/disease & Cases (\%) & Organ/disease & Cases $(\%)$ & Organ/disease & Cases $(\%)$ \\
\hline 1 & Leukemia & 368 (32.7) & Leukemia & $201(33.7)$ & Leukemia & $167(31.5)$ \\
\hline 2 & CNS* & $186(16.5)$ & $\mathrm{CNS}^{*}$ & $98(16.4)$ & $\mathrm{CNS}^{*}$ & $88(16.6)$ \\
\hline 3 & Lymphoma & $88(7.8)$ & Lymphoma & $58(9.7)$ & Gonadal/germ cell & $41(7.7)$ \\
\hline 4 & Gonadal/germ cell & $76(6.8)$ & $\mathrm{SNS}^{\dagger}$ & $41(6.9)$ & Bone tumor & $33(6.2)$ \\
\hline 5 & $\mathrm{SNS}^{\dagger}$ & $69(6.1)$ & Gonadal/germ cell & $35(5.9)$ & Renal tumor & $33(6.2)$ \\
\hline 6 & Bone tumor & $60(5.3)$ & Soft tissue sarcoma & $33(5.5)$ & Lymphoma & $30(5.7)$ \\
\hline 7 & Soft tissue sarcoma & $59(5.2)$ & Bone tumor & $27(4.5)$ & $\mathrm{SNS}^{\dagger}$ & $28(5.3)$ \\
\hline 8 & Renal tumor & $56(5.0)$ & Renal tumor & $23(3.9)$ & Soft tissue sarcoma & $26(4.9)$ \\
\hline 9 & Hepatic tumor & $34(3.0)$ & Hepatic tumor & $20(3.4)$ & Hepatic tumor & $14(2.6)$ \\
\hline 10 & Retinoblastoma & $24(2.1)$ & Retinoblastoma & $14(2.3)$ & Retinoblastoma & $10(1.9)$ \\
\hline Etc. & & $106(9.4)$ & & $46(7.7)$ & & $60(11.3)$ \\
\hline
\end{tabular}

${ }^{*}$ Central nervous system, ${ }^{\dagger}$ Sympathetic nervous system

should be carefully interpreted considering the general population characteristics (e.g. age, sex) and the medical service utilization in each area. In all areas, the relative frequencies of males $(54.0 \sim 61.8 \%)$ were higher than those of females.

\section{3) Distribution of diagnosis methods for malignant neoplasms by gender (Table 3)}

In general, microscopic examination $(82.1 \%)$ was the primary method of diagnosis, followed by clinical investigation (15.2\%), clinical findings only $(2.1 \%)$, specific biochemical/ immunologic tests $(0.5 \%)$, and exploratory surgery $(0.2 \%)$. Eighteen percent of the cancers were diagnosed by clinical judgement alone without having microscopic examination.

\section{4) Frequency of topography and morphology in pri-} mary cancer sites (Table 4)

Table 4 shows the marginal frequencies of topography and morphology in leading malignancies. The most common morphologies (M-code) according to the order of their marginal frequency were adenocarcinomas $(814 \sim 838)$ [37,494 (44.7\%)], papillary \& squamous cell neoplasms $(805 \sim 808) \quad[12,807$ $(15.3 \%)]$, ductal, lobular \& medullary neoplasms $(850 \sim 854)$ $[4,890(5.8 \%)]$, cystic, mucinous \& serous neoplasms $(844 \sim$ 849) $[4,700(5.6 \%)]$, epithelial neoplasms, NOS (801 804) $[3,166(3.8 \%)]$, transitional cell carcinomas $(812 \sim 813)[2,133$ (2.5\%)], Hodgkin's \& non-Hodgkin's lymphomas (959 970) [1,802 (2.2\%)] and leukemias (980 994) [1,686 (2.0\%)]. There were 10,438 cases of malignancies not diagnosed morphologically (M-8000) (12.4\%), similar with the previous year's number [10,655 cases, $12.9 \%]$.

\section{5) Distribution of childhood malignancies by sex and primary sites (Table 5)}

The frequency childhood malignancies, defined as children under the age of 15 , were 1,126 cases (1.3\%). There were 596 male cases and 530 female cases, a sex ratio of 1.1. Leukemia was the most common malignancy $(32.7 \%)$, with a sex ratio of 1.2 , of which subtypes in their order of frequency were ALL (62.5\%), acute non-lymphocytic leukemia (27.2\%), and CML

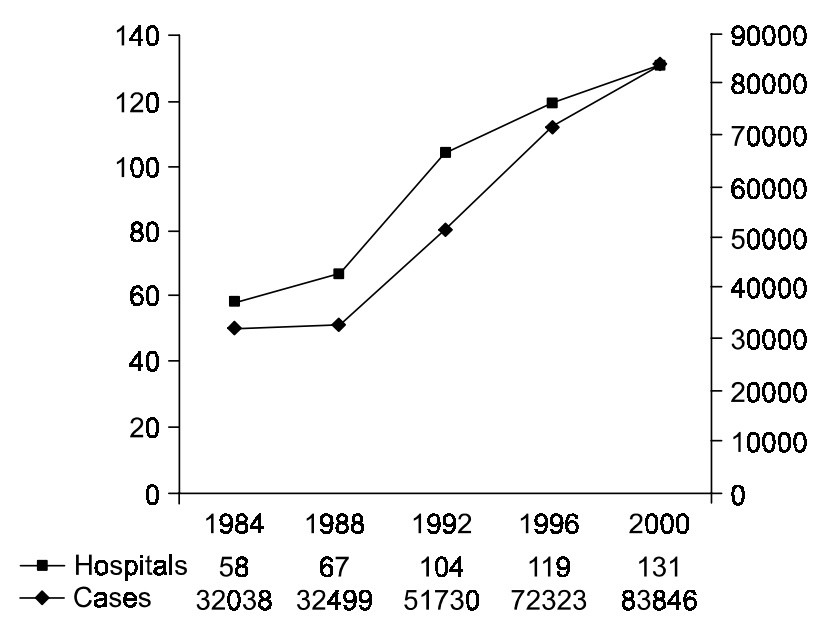

Fig. 2. Numbers of registered hospitals and cases.

(5.2\%). Following leukemia in order of frequency were CNS tumors (16.5\%), lymphomas $(7.8 \%)$, gonadal \& germ cell tumors $(6.7 \%)$, tumors of the sympathetic nervous system $(6.1 \%)$, malignant bone tumors $(5.3 \%)$, soft tissue sarcomas $(5.2 \%)$, renal tumors $(5.0 \%)$, carcinomas and epithelial neoplasms (3.9\%), hepatic tumors (3.0\%), and retinoblastomas $(2.1 \%)$.

\section{DISCUSSION}

Because the Ministry of Health and Welfare required that all University and training hospitals join the KCCR program, the number of affiliated hospitals, and therefore of registered cases of malignancies, have increased year by year (Fig. 2). Because of this fact, the KCCR database is recognized to contain about $80 \%$ of all true cancer cases and to include about $96 \%$ of all cancer patients that visited the KCCR-affiliated hospitals (4).

The KCCR database is especially useful for exhibiting trends 


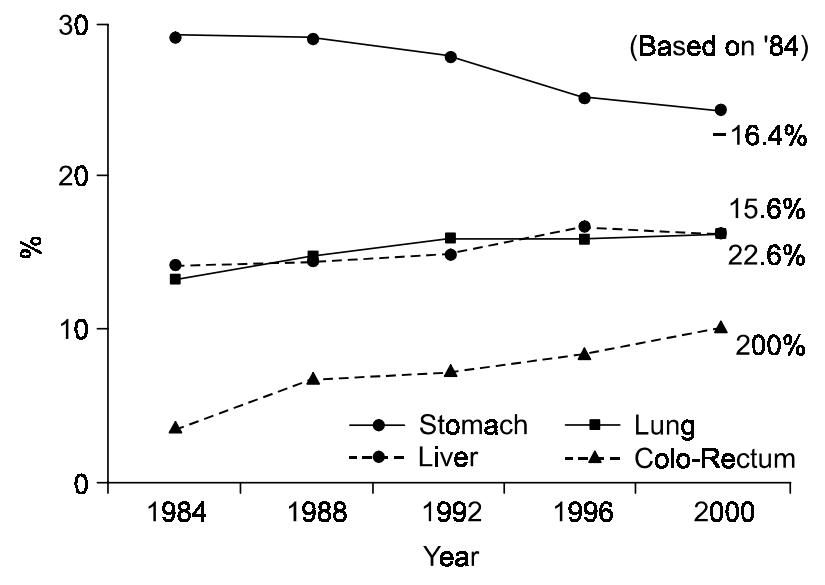

Fig. 3. Trends of the registry in KCCR program by cancer sites in Korean males.

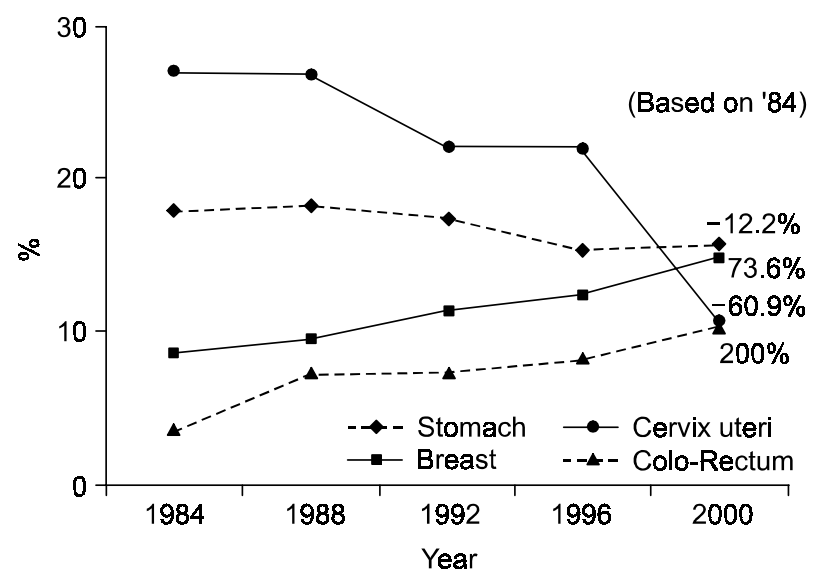

Fig. 4. Trends of the registry in KCCR program by cancer sites in Korean females.

[Appendix] List of the hospitals participating the KCCR program, 2000

Ajou University Hospital

Andong General Hospital

Andong Presbyterian Hospital

Asan Foundation Kangnung Hospital

Asan Medical Center

Bag Ae Hospital

Benedict Hospital

Bupyeong Serim General Hospital

Busan Veterans Hospital

Carollo Hospital

Catholic GEN Hospital

Changwon General Hospital

ChonBuk National University Hospital

Chongji St. Mary's Hospital

Chonnam University Hospital

Choon Hae Hospital

Chosun University Hospital

Chung-Ang University Hospital

ChungAng University Medical Hospital

Chungbuk National University Hospital

Chungnam National University Hospital

College of Medicine, Pochon CHA university CHA General Hospital

Dae Dong Hospital

Dae Jeon Veterans Hospital

Dae Rim St. Mary's Hospital

Daegu Madical Center

DanKook University Hospital

Dong Kang General Hospital

Dong Kang General Hospital

Dong Rae Bong Seng Hospital

Dong-A University Hospital

Dongeui Medical Center

Dongguk University Kyong Ju Hospital

Dong-In Hospital

Dong-Kuk University Po-Hang Hospital

Ewha Womans University Mokdong Hospital

Ewha Womans University Tongdaemun Hospital
Gachon Medical College Gil Medical Center

Gyeongsang University Hospital

Hae Dong Hospital

Hallym Sacred Heart Hospital

Hallym University Hang Gang Sacred Heart Hospital

Hallym University Medical Center ChunCheon Sacred Heart Hospital Hangdong University Sunlin Presbyterian Hospital

Hanil Hospital

Hanyang University Hospital

Hanyang University Kuri Hospital

Holy Hamily Hospital

Il Sin Christian Hospital

In Je University, Pusan Paik Hospital

Inchon Christian Hospital

Inchon Red Cross Hospital

Inchon Choongang General Hospital

Inha University Hospital

Inha University Medical Center Inha Hospital

Inje University Sanggye Paik Hospital

Inje University, Seoul Paik Hospital

Kang Dong Sacred Heart Hospital

Kangbuk Samsung Hospital

Kangnam General Hospital Public Corporation

KangNam Sacred Heart Hospital Hallym University

Keimyung University Medical Center

Koje Hospital

Kon Kuk University Medical Center Seoul Hospital

Konyang University Hospital

Korea Center Center Hospital

Korea University Ansan Hospital

Korea University College of Medical, Guro Hospital

Korea University Hospital

Korea Veterans Hospital

Kosin University Gospel Hospital

Kumi CHA Medical Center

Kwak's Hospital

Kwang Ju Christian Hospital 


\section{[Appendix] Continued}

Kwang Ju Veterans Hospital

Kwang Myung Sung Ae General Hospital

Kwanghye Geneal Hospital

Kyung Hee Medical Center

Kyungpook National University Hospital

Local Public Corp. Inchun Medical Center

Local Public Corporation Chung Cheong Nam-Do Hong Sung Medical Center

Maryknool General Hospital

Masal Samsung Hospital

Metro Hospital

Missionary Benedictine Sisters Fatima Hospital

Mokpo Catholic Hospital

Nam Kwang Hospital

National Health Inaurance Corporation Ilsan Hospital

National Medical Center

National Police Hospital

Pohang St. Mary's Hospital

Presbyterian Medical Center

Pundang Je-Saeng Hospital

Pusan Adventist Hospital

Pusan Medical Center

Pusan National University Hospital

Samsun Hospital

Samsung Medical Center

Sansung Cheil Hospital \& Women's Health Care Center

Sejong General Hospital

Seo San Medical Center

Seoul Adventist Hospital

Seoul Municipal Boramea Hospital
Seoul National University Hospital

Seoul Red Cross Hospital

Severance Hospital Yonsei University Medical College

Shin Chun Hospital

Soonchunhyang GuMi Hospital

Soonchunhyang University Chonam Hospital

Soonchunhyang University Hospital

St, Mary's Hospital, The Catholic University of Korea

St, Francisco General Hospital

St, Paul's Hospital

Sun General Hospital

Sung Ae Hospital

Taeback Choongang General Hospital

Taegu Catholic University Hospital

Taegu Fatima Hospital

Taegu Verterans Hospital

Tae-jeon Eul-ji Cellege Hospital

The Catholic University of Korea Kangnam St. Mary's University

The Catholic University of Korea Taejon St. Mary's University

The Catholic University of Korea, Our Lady of mercy University

The Catholic University of Korea, St. Vincent's Mary's University

Uijongbu St. Mary's Hospital The Catholic University Korea

Ulsan University Hospital

Wallace Memorial Baptist Hospital

Wonju Christian Hospital, Won Ju Clooege of Medicine, Yonsei University Wonkwang University Hospital

Yeungnam University Medical Center

Yonsei Medical Center, Yong-Dong Severance Hospital

Young Dong Hospital of cancer occurrence in Korea because information on thechanging trends of cancer incidence over time is an essential component in the planning and monitoring of programs for cancer prevention, early detection and treatment. For example, while stomach cancer has shown a decreasing trend in both Korean males and females since 1980s, the incidence of lung cancer is rapidly increasing, especially in males (Fig. 3, 4). The confirmation of this trend suggests the necessity of introducing some control programs.

The one of the limitations of the KCCR data was the impossibility of estimating the prevalence rate because only incident cases that followed the registration protocol were reported. Moreover, being a nationwide hospital-based cancer registry, the KCCR data could not provide the true incidence rate. To overcome these limitations, some population-based, regional cancer registry programs were conducted as an ongoing part of this KCCR program in December 2001 in 8 areas, Seoul, Busan, Daegu, Incheon, Gwangju, Daejeon, Ulsan, and Jejudo. The KCCR database will continue to hold the most important position until a nationwide, population-based, data acquisition program is constructed on the basis of international standards.

One caution in the interpretation of the KCCR data is that the numbers represent cases rather than patients. The patients with distinct cancers at several sites were treated as multiple primary patients and reported according to the cancer site. A further caution in the interpretation of this data is that some registered patients in one annual report might also be registered in another annual report. This possibility necessitates the keeping of comparisons of annual reports of cases in which there was a delay in registration. To resolve this problem, we gathered annual report data for 5 years and have published two 5 -year reports $(5,6)$. We have also planned to report all registered cases.

\section{ACKNOWLEDGEMENTS}

We would like to acknowledge the medical record administrators in the KCCR-affiliated hospitals [Appendix] whose enthusiastic participation in the KCCR program, 2000.

\section{REFERENCES}

1. Ahn YO. Population-based cancer registries in Korea. Asian Pacific J Cancer Prevention 2001;(Suppl. Volume 2):39-42.

2. Bae JM, Won YJ, Jung KW, Suh KA, Ahn DH, Park JG. Annual Report of the Central Cancer Registry in Korea 1999: based on registered data from 128 hospitals. Cancer Research and Treatment 2001;33(5):367-372.

3. Ministry of Health and Welfare, Korea. Annual report of Korea Central Cancer Registry program, January $\sim$ December, 2000. 
Korea, 2002. Available from URL: http://www.ncc.re.kr 4. Bae JM, Won YJ, Jung KW. The survey for construction and completeness of the databases of Korea Central Cancer program. National Cancer Center: Korea: 2001. (in Korean) 5. Ministry of Health and Welfare, Korea. Cancer registry pro- gram in the Republic of Korea, 1982-1987 (I,II). Korea: 1988. (in Korean)

6. Ministry of Health and Welfare, Korea. Cancer registry program in the Republic of Korea, 1988-1992. Korea: 1995. (in Korean) 